\title{
ESTUDO ESPECTROSCÓPICO DO EQUILÍBRIO ENTRE ESPÉCIES HEXACOORDENADA E PENTACOORDENADA DOS MONÔMERO $d$ NATIVO E RECONSTITUÍDO DA HEMOGLOBINA EXTRACELULAR GIGANTE DE Glossoscolex paulistus EM MEIO ALCALINO
}

\author{
Julio C. Ribelatto*, Alessandra L. Poli, Leonardo M. Moreira e Hidetake Imasato \\ Instituto de Química de São Carlos, Universidade de São Paulo, CP 780, 13560-970 São Carlos - SP, Brasil
}

Recebido em 18/3/05; aceito em 16/9/05; publicado na web em 14/3/06

\begin{abstract}
SPECTROSCOPIC STUDY OF THE EQUILIBRIUM BETWEEN HEXACOORDINATED AND PENTACOORDINATED SPECIES OF THE NATIVE AND RECONSTITUTED $d$ MONOMERS OF THE Glossoscolex paulistus GIANT EXTRACELLULAR HEMOGLOBIN IN ALKALINE MEDIUM. UV-Vis and fluorescence spectroscopic studies of native and reconstituted $d$ monomers from Glossoscolex paulistus were performed in alkaline $\mathrm{pH}$. In addition to the presence of aquomet and hemichrome hexacoordinated species, a pentacoordinated species occurs. This latter species, which presents an imidazolate group of axial histidine as fifth ligand, is identified by the blue-shifted low-intensity Soret band and LMCT band. The pentacoordinated species must occur due to partial polypeptide unfolding.
\end{abstract}

Keywords: extracellular hemoglobin; hexacoordinated; pentacoordinated.

\section{INTRODUÇÃO}

O anelídeo Glossoscolex paulistus é um animal pertencente ao filo Anelidae, à classe Oligochaeta e à família Glossoscolecidae ${ }^{1}$. Esta minhoca gigante, popularmente conhecida como minhocoçu, é encontrada em determinados sítios das cidades de Piracicaba, Araras e Rio Claro, no estado de São Paulo, Brasil2 ${ }^{2}$, tendo solos calcários como seu habitat natural ${ }^{1}$. Este anelídeo, que pertence a uma classe muito empregada em estudos ecológicos de solos ${ }^{3}$, apresenta comprimento que varia de 280 a $305 \mathrm{~mm}$, com o maior diâmetro na região mediana do corpo alcançando de 10 a $11 \mathrm{~mm}^{2}$.

A hemoglobina $(\mathrm{Hb})$ extracelular gigante de G. paulistus é uma hemoproteína oligomérica ${ }^{4}$ com massa molecular em torno de 3,1 $\mathrm{MDa}^{5}$, apresentando ponto isoelétrico ácido ${ }^{6}$. A hemoglobina de G. paulistus pertence à mesma classe da hemoglobina do anelídeo Lumbricus terrestris, que tem sido intensamente investigada ${ }^{7-13}$, apesar de ainda haver algumas controvérsias quanto à sua massa molecular ${ }^{14}$. Vale lembrar que essa classe de hemoglobinas extracelulares gigantes, também chamadas eritrocruorinas, representa, segundo Vinogradov ${ }^{6}$, o ápice de complexidade em hemoproteínas que carregam oxigênio. Ademais, estas hemoglobinas são consideradas altamente cooperativas ${ }^{15}$ e extremamente interessantes do ponto de vista da seleção natural, uma vez que trabalhos asseveram características não usuais de adaptação em meios ricos em sulfetos, por ex. ${ }^{16}$.

A hemoglobina de L. terrestris tem, inclusive, sido testada como sangue artificial em função da sua natureza extracelular, do seu grande tamanho e da sua resistência à oxidação ${ }^{17-19}$. De fato, Hirsch e colaboradores ${ }^{17}$ asseveraram que a hemoglobina de L. terrestris apresenta, em meio neutro, afinidade por oxigênio e cooperatividade semelhantes à hemoglobina humana. Ademais, as hemoglobinas extracelulares gigantes, inclusive a hemoglobina de G. paulistus ${ }^{18,20}$, não apresentam a mesma predisposição das hemoglobinas tetraméricas para se dissociarem quando diluídas ${ }^{18,20}$. Inclusive, é esperado que as hemoglobinas extracelulares gigantes sejam mais baratas, mais fáceis de se estocar e menos suscetíveis de causarem

*e-mail: ribelatto@iqsc.usp.br respostas imunológicas ${ }^{18}$. Essa menor ação em relação ao sistema imunológico ocorre por se tratar de uma hemoglobina extracelular, ou seja, não se encontra dentro de uma célula sanguínea, não tendo, portanto, sua presença associada concomitantemente à ocorrência das membranas plasmáticas inerentes às células, que são estruturas que induzem respostas imunológicas.

A estrutura da hemoglobina de G. paulistus apresenta 144 globinas e 36 cadeias polipeptídicas sem o grupo prostético heme, que são cruciais para sua integridade estrutural (cadeias "linkers") ${ }^{21}$, sendo que o arranjo da hemoglobina íntegra se dispõe espacialmente como 2 discos hexagonais sobrepostos, com as subunidades estabilizadoras, "linkers", na porção central desse arranjo oligomérico $^{21}$. De fato, em estudos com a hemoglobina de $L$. terrestris é proposta uma distribuição assimétrica para essas cadeias "linkers", o que estaria associado ao grande momento de dipolo apresentado pelo arranjo supramolecular ${ }^{6}$.

As 144 globinas estão organizadas como 12 dodecâmeros, sendo que cada dodecâmero consiste em um conjunto de três tetrâmeros. Cada tetrâmero, por sua vez, é constituído por três trímeros (cadeias monoméricas $a, b, c$ unidas covalentemente através de ligações dissulfeto) e uma cadeia monomérica $d^{11,22}$.

No presente trabalho, o monômero $d$ foi isolado, em $\mathrm{pH} 9,0$, através de um método de separação cromatográfica baseado na filtração em gel, visando a caracterização das espécies presentes no equilíbrio em meio alcalino. É importante mencionar que o estudo de uma cadeia única pode ser altamente relevante para a compreensão da hemoglobina como um todo devido à maior simplicidade dessa abordagem, já que pode haver diferenciação de propriedades entre os grupos heme do tetrâmero. Ademais, em se tratando de um estudo de reconstituição, não seria trivial uma abordagem que envolvesse um arranjo que apresenta 144 hemes, pois seria extremamente complexo garantir que todas as 144 globinas foram reconstituídas.

Deste modo, a proposta deste estudo é avaliar os equilíbrios químicos das espécies formadas na primeira esfera de coordenação do centro férrico do monômero $d$ em função do $\mathrm{pH}$, em meio alcalino, uma vez que o trabalho anterior ${ }^{23}$ foi desenvolvido em meio ácido. Esta análise pode apresentar correlações com os me- 
canismos de desenovelamento polipeptídico da globina, principalmente em meios mais drásticos para a proteína, como por ex., valores extremos de pH. Segundo Marques e Brown ${ }^{24}$, o fato da porfirina desenvolver diversas funções no sistema biológico é uma clara evidência da influência efetiva do ambiente protéico em suas propriedades. Assim sendo, correlacionar modificações que ocorrem no grupamento prostético com aquelas que acontecem na cadeia polipeptídica propriamente considerada representa contribuição relevante para a compreensão da relação estrutura-atividade das hemoglobinas. Por conseqüência, definir os ligantes axiais, assim como o estado de spin do sistema ferro-porfirínico, pode fornecer informações específicas sobre os processos de desnaturação desta hemoglobina gigante extracelular. De fato, estados pentacoordenados ${ }^{25,26} \mathrm{e}$, até mesmo, tetracoordenados ${ }^{27,28}$, podem, em alguns casos, ser indicativos de significativa perda das estruturas secundária e terciária da hemoproteína.

Por outro lado, em hemoglobinas tetraméricas, a formação das espécies hexacoordenadas conhecidas como hemicromo, ou seja, hemes ligados axialmente por dois resíduos de aminoácidos, é atribuída, em grande parte, às hemoglobinas desnaturadas ${ }^{29,30}$. Na maioria das hemoproteínas, as histidinas são os resíduos que se ligam ao centro metálico axialmente, isto é, nas posições proximal e distal, constituindo, conseqüentemente, formas férricas baixo-spin. A ocorrência dos hemicromos em hemoglobinas tetraméricas está associada à formação de corpos de Heinz e ao processo de envelhecimento celular das hemácias ${ }^{29}$. Entretanto, outros estudos enfatizam que, apesar da forte correlação entre a transição de aquometa para hemicromo com o aumento da solubilidade do heme, a formação do hemicromo não necessariamente significaria um processo de desnaturação propriamente dito. De fato, sabendo-se que alguns trabalhos correlacionam a formação de hemicromo à ocorrência de determinados canais de acesso ao solvente ${ }^{31}$, este fenômeno poderia ser, a priori, simplesmente uma mudança conformacional, sem uma perda mais expressiva das estruturas secundária e terciária da proteína. Mesmo em trabalhos envolvendo as duas mais novas globinas descobertas em vertebrados, neuroglobina e citoglobina, a formação de hemicromos e seu estudo espectroscópico tem sido de grande importância, visando a compreensão das propriedades dessas hemoproteínas ${ }^{32,33}$. Neste sentido, vale lembrar que Blumberg e Peisach consideram a transição de spin que leva à formação do hemicromo como uma conseqüência da quebra de ligações hidrofóbicas ${ }^{34}$, o que implicaria, de qualquer maneira, em um maior ou menor rearranjo, enfatizando, uma vez mais, a correlação entre mudanças na primeira esfera de coordenação e alterações conformacionais das cadeias polipeptídicas.

Uma propriedade característica dos hemes em sistemas protéicos é a presença de configurações eletrônicas baixo-spin com níveis de energia muito próximos uns dos outros. Por causa desta pequena separação de energia entre os estados de spin, as conformações do heme e suas propriedades eletrônicas são muito sensíveis ao número, à natureza e orientação dos ligantes axiais coordenados ao centro férrico ${ }^{35-40}$. A proximidade dos diferentes estados de spin pode ser essencial à função protéica. Ademais, segundo Marques e Brown ${ }^{24}$, um dos mais significativos desenvolvimentos na química de porfirinas nos últimos 20 anos tem sido o estudo sobre a extraordinária flexibilidade do anel porfirínico ${ }^{39,40}$, que está profundamente associada aos fatores supracitados.

Dentro deste contexto, a presente abordagem faz uso, não somente do monômero $d$ nativo, mas também do monômero $d$ reconstituído, tendo por escopo a compreensão mais ampla das correlações intrínsecas entre os fenômenos que ocorrem no heme e aqueles que acontecem no enovelamento polipeptídico. Afinal, há reconstituições que perturbam a estrutura terciária ou alteram a afinidade do centro metálico pelo sexto ligante axial, apesar destas ocorrências não constituírem regra para todas as hemoproteínas.

Este trabalho consiste em uma pesquisa espectroscópica comparativa dos monômeros $d$ nativo e reconstituído da hemoglobina extracelular gigante de G. paulistus, com enfoque especial na identificação das espécies que coexistem em cada valor de $\mathrm{pH}$, uma vez que o estudo da complexidade do equilíbrio das hemoproteínas em função do $\mathrm{pH}$ pode representar grande avanço na compreensão da relação estrutura-atividade destes sistemas bioinorgânicos supramoleculares.

\section{PARTE EXPERIMENTAL}

\section{Obtenção e purificação: oxi-hemoglobina íntegra, meta- hemoglobina e monômero}

A hemolinfa foi extraída com pipetas de Pasteur em presença de anti-coagulante (citrato de sódio (Baker) 0,1 mM) e armazenada em banho gelado durante a coleta. A primeira etapa da purificação foi realizada através de centrifugação da hemolinfa a 15000 rpm por 15 min a $4{ }^{\circ} \mathrm{C}$ para precipitação de impurezas. Em seguida, realizou-se uma diálise contra tampão TRIS-HCl (Merck) 100 mM, pH 7,0 com 0,1 mM EDTA (Sigma). Após a diálise, efetuouse a ultracentrifugação da proteína a $4{ }^{\circ} \mathrm{C}$ por $6 \mathrm{~h}$ a $35000 \mathrm{rpm} \mathrm{e}$, em seguida, promoveu-se nova suspensão em tampão TRIS-HCl $100 \mathrm{mM}, \mathrm{pH}$ 7,0 com 0,1 mM EDTA, com posterior purificação por cromatografia de exclusão em coluna de gel Sephadex G-200 equilibrada com tampão TRIS-HCl $100 \mathrm{mM}, \mathrm{pH}$ 7,0 com $0,1 \mathrm{mM}$ EDTA. Devido à estabilidade da estrutura oligomérica em pH 7,0, o eluato coletado no volume de exclusão contém a oxiHb íntegra pura, sendo estocado a $4{ }^{\circ} \mathrm{C}$ para uso posterior ${ }^{41}$. A fração oximonômero foi separada eluindo-se a oxiHb íntegra estabilizada em pH 9,0 em uma coluna de gel Sephadex G-200 nas mesmas condições apresentadas acima. A meta-hemoglobina foi obtida através de uma reação de oxidação da oxiHb íntegra com ferricianeto de potássio, $\mathrm{K}_{3}\left[\mathrm{Fe}\left(\mathrm{CN}_{6}\right)\right]$, na razão molar de 3:1 em relação à concentração de heme $e^{11,42}$. A fração meta-monômero foi separada aplicando-se a meta-hemoglobina estabilizada em pH 9,0 em uma coluna de gel Sephadex G-200 pelo mesmo procedimento.

\section{Obtenção do apomonômero}

O apomonômero foi isolado a partir da forma oxi-monômero separada por cromatografia de exclusão em $\mathrm{pH}$ 9,0 como descrito acima. O grupo heme foi extraído com metiletilcetona (Ecibra) após ajustar-se o $\mathrm{pH}$ para 3,8, consoante metodologia estabelecida por Teale ${ }^{43}$

\section{Reconstituição do monômero}

A solução de hemina (Sigma) foi preparada empregando-se um volume mínimo de $\mathrm{NaOH}$ (Merck) 0,2 M obtendo-se um estoque de $0,153 \mu \mathrm{M}$. A reconstituição foi realizada a partir do contato entre a solução de hemina com alíquotas da solução de globina (apomonômero) em tampão TRIS-HCl $20 \mathrm{mM}, \mathrm{pH}$ 7,0, contendo $1 \mathrm{mM}$ de EDTA.

\section{Medidas de absorção eletrônica}

Os espectros de absorção eletrônica foram medidos entre 250700 nm, em um aparelho Shimadzu UV-1601 PC, à temperatura ambiente. Os espectros foram armazenados com o objetivo de serem posteriormente analisados empregando-se o programa CCA 
("convex constraint analysis"), de Fasman e colaboradores ${ }^{44}$. O programa CCA permite calcular os espectros das espécies químicas que coexistem em solução, ou seja, os espectros que são denominados de espectros bases, através da análise do conjunto dos espectros experimentais, que são as funções iniciais. Um espectro de absorção em uma dada condição experimental pode ser representado pela função $A(\lambda)$ :

$A(\lambda)=\sum_{i=1}^{P} a_{i} A_{i}(\lambda)$

nesta Equação, $a_{i}$ é o coeficiente que determina a proporção do constituinte $i ; A_{i}(\lambda)$ são os espectros das soluções das espécies químicas $i$, constituindo-se nos espectros bases; $P$ é o número total de espécies químicas em solução. As restrições impostas pelo programa são:

1) $\sum_{i=1}^{P} a_{i}=1$; 2) $a_{i} \geq 0$; 3) $A_{i}$ 's são linearmente independentes

A partir das Figuras 1(c) e 2(c) foi possível calcular os valores de pK para os monômeros $d$ nativo e reconstituído pelo ajuste através da Equação abaixo:

$H A \Leftrightarrow H^{+}+A^{-}$

$\mathrm{C}_{0}(1-f) . .\left[\mathrm{H}^{+}\right] \mathrm{C}_{0} f$

$K=\frac{\left[H^{+}\right] C_{0} f}{C_{0}(1-f)}$

$f=\frac{K}{\left[H^{+}\right]+K}$

\section{Medidas de fluorescência}

As medidas da emissão de fluorescência dos monômero nativo e reconstituído $d$ da $\mathrm{Hb}$ de $G$. paulistus na forma meta foram realizadas em um aparelho Hitachi F-4500, em meio alcalino, variando-se o pH de 7,0 a 12,0. Essa emissão fluorescente é oriunda dos três resíduos de triptofano presentes na cadeia monomérica $d$. $\mathrm{O}$ comprimento de onda de excitação foi de $295 \mathrm{~nm}$ e o espectro de emissão acompanhado entre 305 a $450 \mathrm{~nm}$. As amostras foram preparadas com absorbância em $295 \mathrm{~nm}\left(\mathrm{~A}_{295}\right) \leq$ a 0,1 , com o objetivo de se evitar os efeitos de absorção interna, e todos os resultados foram normalizados para um valor de $\mathrm{A}_{295}=0,1$.

\section{RESULTADOS E DISCUSSÃO}

Estudo por absorção eletrônica e emissão de fluorescência em função do pH

\section{Absorção eletrônica}

Os espectros de absorção na região do ultravioleta-visível dos monômeros $d$ nativo e reconstituído da meta-hemoglobina de $G$. paulistus foram obtidos na região entre 250 e $700 \mathrm{~nm}$, em função do $\mathrm{pH}$.

Na Figura 1a são apresentados os espectros óticos do monômero $d$ nativo e na Figura 2a são mostrados os espectros do monômero $d$ reconstituído na faixa de $\mathrm{pH}$ entre 7,0 e 12,0. Em pH 7,0, para ambos os casos, a banda de Soret está centrada em $413 \mathrm{~nm}$ e as bandas $\beta$ e $\alpha$ possuem máximos, respectivamente, em 535 e $565 \mathrm{~nm}$, constituindo, por conseguinte, espectros característicos de hemicromo ${ }^{34,45-48}$. Isto indica que o método de separação cromatográfica em pH 9,0 gerou as condições para a transição da
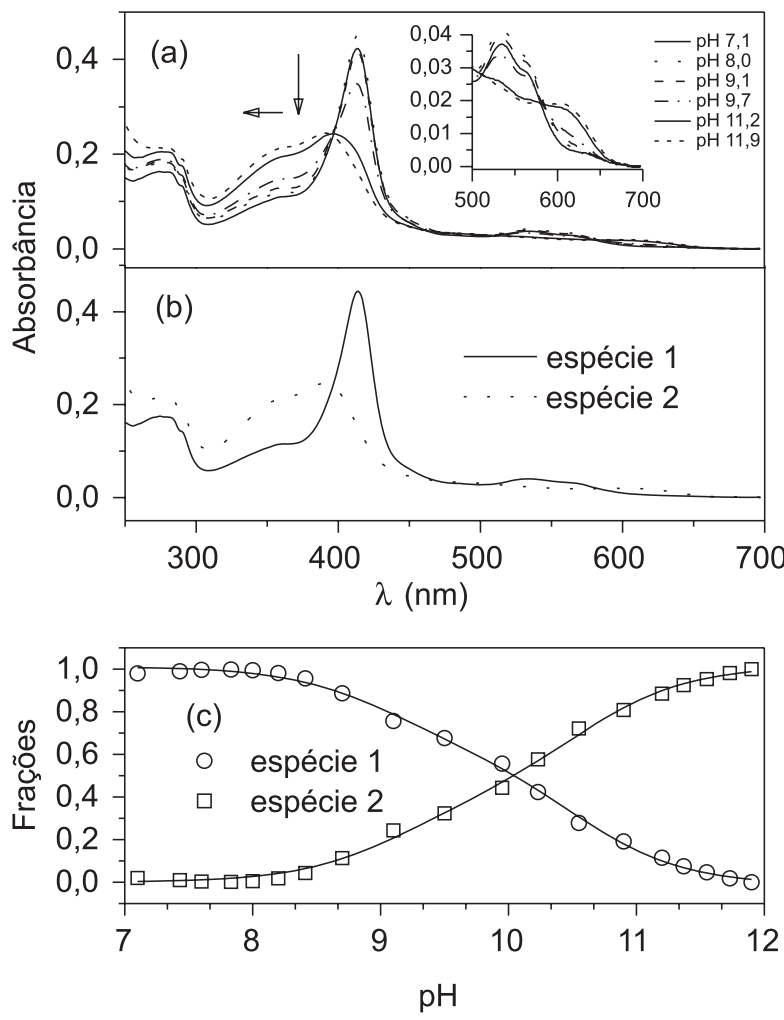

Figura 1. (a) Espectros eletrônicos do monômero d nativo da metaHb de G. paulistus em função do $\mathrm{pH}$ através de titulação; (b) espécies predominantes (hemicromo - espécie 1 / espécie pentacoordenada - espécie 2) correspondentes aos componentes dos espectros experimentais em diferentes proporções, em função do $\mathrm{pH}$; (c) porcentagem das frações de cada espécie em função do $\mathrm{pH}$. Ajustes da fração de cada uma das espécies presentes no equilíbrio. $O$ inserto em la é uma ampliação das bandas $Q$

espécie aquometa para a espécie hemicromo, que consiste em um complexo bis-histidina. Por conseguinte, nota-se que esta transição tem, no mínimo, uma irreversibilidade parcial, uma vez que o retorno para $\mathrm{pH}$ 7,0 pouco regenera a espécie inicial aquometa.

Com a subseqüente alcalinização, evidencia-se a ocorrência de uma diminuição da intensidade da banda de Soret com concomitante deslocamento do máximo para $390 \mathrm{~nm}$ e o surgimento de um ombro em $360 \mathrm{~nm}$ (Figura 1a), o que constitui um comportamento característico da formação de uma espécie pentacoordenada. Visca e colaboradores ${ }^{49}$ empregam, inclusive, a determinação da razão entre as intensidades das bandas de Soret do composto estudado e de uma espécie atribuída como hexacoordenada para determinar a presença da espécie pentacoordenada, uma vez que este estado de coordenação tem uma absortividade molar muito menos intensa. De qualquer maneira, os dois fenômenos espectrais acima discutidos, ou seja, o deslocamento hipsocrômico e a diminuição de absortividade molar são fortes indicativos da formação de uma espécie pentacoordenada, conforme uma série de trabalhos envolvendo hemoproteínas, por meio de uma variada série de técnicas espectroscópicas ${ }^{27,28,50-61}$. Ademais, surge uma banda de transferência de carga do ligante para o metal (TCLM) em 620 nm, que também corresponde a uma espécie de spin alto, como é o caso do pentacoordenado.

Harris e Gilda ${ }^{62}$ desenvolveram cálculos quânticos semiempíricos a respeito da estrutura eletrônica do espectro de absorção na região do ultravioleta-visível dos estados baixo e alto spin de citocromo P450 e afirmaram que a transição de baixo para alto 
spin implica em um deslocamento para o azul da banda de Soret. O estado baixo spin apresentaria mistura acentuada dos orbitais $d$ (e e e $\pi^{*}$ da porfirina, resultando em uma diminuição da energia deste orbital $\mathrm{e}_{\mathrm{g}}-\pi^{*}$ e um conseqüente deslocamento para o vermelho da banda de Soret. Este fenômeno só poderia ocorrer se o anel porfirínico estivesse ao menos ligeiramente distorcido, pois do contrário não haveria superposição dos orbitais $d\left(\mathrm{e}_{\mathrm{g}}\right)$ de simetria $\sigma$ (sigma) com os orbitais $\pi^{*}$. De qualquer maneira, a distorção do anel porfirínico é comum em hemoproteínas em função de uma série de interações estéricas do heme com o bolsão hidrofóbico polipeptídico. Como os orbitais $d\left(\mathrm{e}_{\mathrm{g}}\right)$ não estão preenchidos em uma espécie baixo spin, o orbital ligante originado da combinação deste orbital $d\left(\mathrm{e}_{\mathrm{g}}\right)$ com o orbital $\pi^{*}$ do anel porfirínico seria um novo orbital de energia mais baixa e não preenchido, causando o deslocamento para o vermelho da transição eletrônica de Soret em um complexo baixo spin. Esta proposta parece ser aplicável a outras hemoproteínas, em especial, à hemoglobina de G. paulistus. De fato, para o monômero estudado neste trabalho, a banda de Soret, que representa um sistema baixo spin como o hemicromo (complexo bis-histidina), está deslocada para o vermelho em relação às bandas que representam duas espécies alto spin, que são a forma aquometa e uma forma pentacoordenada.

Provavelmente, o ligante que deixa a primeira esfera de coordenação do centro férrico na formação do heme pentacoordenado seria a histidina distal, devido à maior tensão da sua ligação com o centro férrico em relação à coordenação da histidina proximal, entretanto essa identificação não é trivial e outros estudos espectroscópicos são necessários para se obter maior evidência sobre qual das histidinas é dissociada.

Na Figura 2a, verifica-se comportamento similar para o monômero $d$ reconstituído. Em suma, na faixa de $\mathrm{pH}$ entre 7,0 e 10,0 predomina a espécie de spin baixo (hemicromo) e em valores de $\mathrm{pH}$ acima de 10,0 prevalece a espécie de spin alto, que consiste em uma espécie pentacoordenada, apresentando uma das histidinas como quinto ligante. Nistor e colaboradores ${ }^{63}$, estudando uma neuroglobina férrica através da ressonância paramagnética eletrônica (RPE), propõem que, partindo-se de uma espécie hemicromo em uma transição dependente do $\mathrm{pH}$, ocorreria a formação de uma espécie pentacoordenada com uma histidina na quinta posição de coordenação.

Entretanto, os comprimentos de onda observados para esta espécie pentacoordenada em meio alcalino diferem, significativamente, dos valores encontrados para o monômero $d$ pentacoordenado em meio ácido, conforme descrito em trabalho anterior ${ }^{23}$. É provável que o ligante imidazólico proveniente da histidina distal se encontre desprotonado em função da alta alcalinidade do meio quando ocorre a transição em questão. Desta forma, o quinto ligante do centro férrico não seria o imidazol propriamente dito, mas sim, o grupo imidazolato.

Este fato justificaria uma saída mais pronunciada do íon férrico em relação ao plano dos anéis pirrólicos do heme, haja vista a maior basicidade do imidazolato comparado à sua forma protonada, isto é, o imidazol. Esta proposta também é plausível se for levada em consideração a significativa eletroafinidade do centro metálico em estado de oxidação $3+$, o que causaria uma atração eletrostática muito maior pelo imidazolato, que é um grupo aniônico, que pelo ligante neutro imidazol. Esta saída mais pronunciada do íon férrico causaria o deslocamento hipsocrômico da banda de transferência de carga do ligante para o metal (TCLM) em relação ao pentacoordenado em meio áci$\mathrm{do}^{23}$. De qualquer maneira, Boffi e colaboradores ${ }^{64}$ estabeleceram que $^{2}$ a presença da banda de transferência de carga, $d$ (íon férrico) $\rightarrow \pi$ (heme), tanto em 602 como em $635 \mathrm{~nm}$, indica a presença de complexos de ferro alto-spin, o que corrobora a presente discussão.
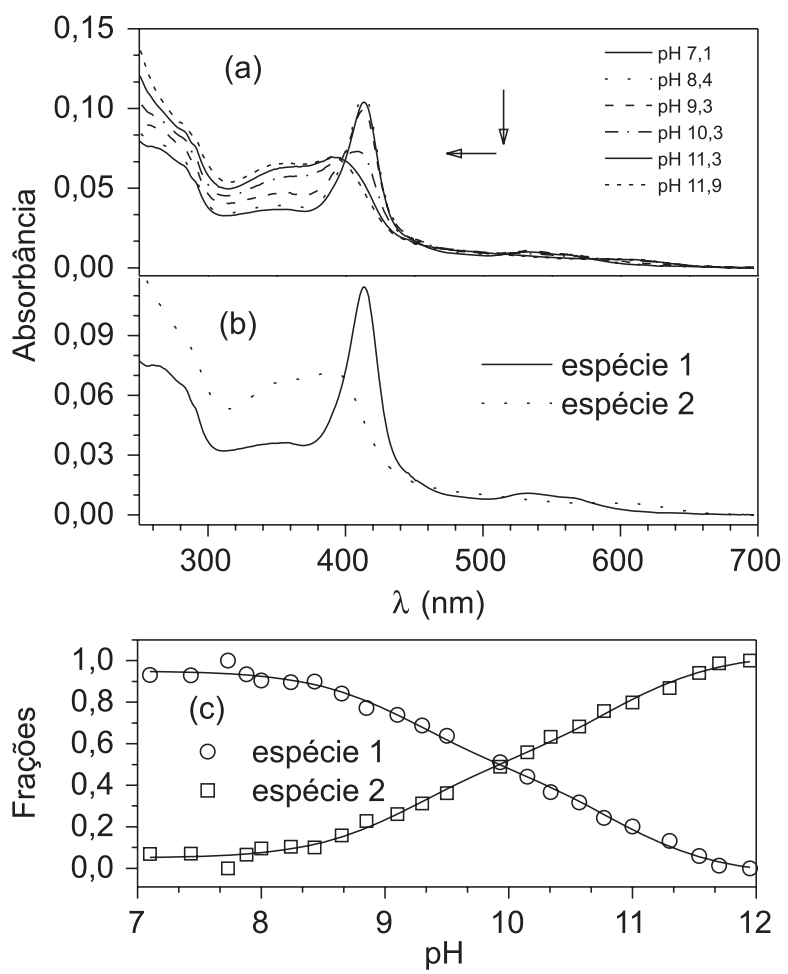

Figura 2. (a) Espectros eletrônicos do monômero d reconstituído da metaHb de G. paulistus em função do pH através de titulação; (b) espécies puras (hemicromo - espécie 1 / espécie pentacoordenada - espécie 2) correspondentes aos componentes dos espectros experimentais em diferentes proporções em função do $\mathrm{pH}$; (c) porcentagem das frações de cada espécie em função do $\mathrm{pH}$. Ajustes da fração de cada uma das espécies presentes no equilíbrio

Este deslocamento hipsocrômico da banda de Soret do heme pentacoordenado alcalino em relação ao pentacoordenado ácido pode ser compreendido considerando-se que no sistema hexacoordenado baixo-spin "puro", a superposição de orbitais entre o centro férrico e os nitrogênios porfirínicos é cerca de três vezes maior que a superposição de orbitais entre o centro férrico e o nitrogênio histidínico, já que as histidinas estão bem mais distantes do metal que a porfirina ${ }^{35}$. Esta diferença de superposição de orbitais faz com que o deslocamento do centro metálico diminua o desdobramento de campo cristalino dos orbitais $d$ em questão, uma vez que, por mais que ocorresse uma superposição significativa com o quinto ligante axial, a perda de interação com os orbitais porfirínicos seria bem mais representativa por se tratar de um ligante tetraazamacrocíclico, ou seja, um ligante tetradentado. Como a saída do íon férrico seria mais pronunciada no meio alcalino, o desdobramento dos orbitais $d$ seria bem menos intenso nestas condições que em meio ácido. A conseqüiente desestabilização dos orbitais $d_{\pi}$ do metal, que são os orbitais que acomodam o elétron proveniente da transição eletrônica de transferência de carga, faria com que essa transição ficasse mais energética, ou seja, com menor comprimento de onda.

Após detectar-se a presença de pontos isosbésticos, conduziuse a análise do conjunto de espectros, utilizando-se o algoritmo $\mathrm{CCA}^{44}$, considerando-se duas espécies presentes em solução. Esta análise proporcionou os espectros representados na Figuras 1(b) e 2(b), que foram atribuídos às duas espécies químicas em solução: hemicromo (espécie de spin baixo: espécie 1) e uma espécie pentacoordenada (espécie de spin alto: espécie 2).

Nas Figuras 1(c) e 2 (c) são apresentadas as frações de cada espécie em função do $\mathrm{pH}$, com valores de pK em torno de 10,0, tanto para o nativo como para o reconstituído. Trabalhos de resso- 
nância paramagnética eletrônica, que estão em andamento, corroboram essas análises, haja vista a intensidade significativa das linhas axiais rômbicas de spin alto na faixa de $\mathrm{pH}$ na qual o pentacoordenado é o componente predominante.

De fato, a observação de uma forte dependência do $\mathrm{pH}$ na formação da espécie pentacoordenada do molusco Aplysia limacina foi aprioristicamente considerada uma consequiência, pelo menos em parte, da ausência da histidina distal. Entretanto, a hemoglobina Scapharca inaequivalvis também mostra uma forte dependência do $\mathrm{pH}$, quando da formação de uma espécie pentacoordenada, mesmo contendo a histidina na posição distal ${ }^{33}$. Esta informação é de grande relevância para o presente trabalho, uma vez que o trabalho de Madura e colaboradores ${ }^{15}$ estabeleceu que as hemoglobinas de L. terrestris e Scapharca inaequivalvis apresentam significativa similaridade em termos de estrutura secundária. De fato, se considerarmos a semelhança entre as hemoglobinas $L$. terrestris e $G$. paulistus seria plausível inferir uma possível tendência única para estas hemoglobinas gigantes extracelulares.

A existência das transições mencionadas acima foi também observada para a hemoglobina íntegra, o que ressalta as excelentes características do monômero $d$ como sistema modelo da hemoglobina em sua condição nativa.

É importante salientar que a formação do hemicromo não necessariamente indica um processo de desnaturação propriamente dito, mas quase sempre uma mudança conformacional mais ou menos pronunciada dependendo da proteína, especialmente envolvendo as hélices E e F, que são aquelas dos sítios axiais do centro metálico ${ }^{65}$. Riccio e colaboradores ${ }^{65}$ propõem, para uma hemoglobina tetramérica, que o hemicromo seria um estado intermediário entre os chamados estados $\mathrm{R}$ (relaxado) e T (tenso), que são associados, por sua vez, a efeitos alostéricos das hemoglobinas. Vale lembrar que se correlaciona o estado $\mathrm{T}$ a uma configuração pentacoordenada e o estado R, a uma configuração hexacoordenada para o centro férrico ${ }^{66}$.

Assim sendo, algo semelhante poderia acontecer com o monômero $d$, ou seja, a formação da espécie hemicromo seria uma etapa intermediária de um processo maior que culminaria na formação da espécie pentacoordenada. Estudos anteriores enfocando a hemoglobina íntegra de G. paulistus já sugeriam que a formação do hemicromo não estaria associada diretamente a um fenômeno de desnaturação ${ }^{67}$. De fato, a formação dos pentacoordenados deve estar associada a um aumento da acessibilidade do solvente aquoso no bolsão porfirínico. Sabendo-se que o presente estudo avalia o comportamento de uma única cadeia isolada, é possível inferir a influência do solvente aquoso na troca de ligantes do grupo heme da hemoglobina de $G$. paulistus, sem a influência de quaisquer efeitos alostéricos provenientes de outras cadeias polipeptídicas.

É possível que a principal diferença entre o monômero isolado $d$ e a hemoglobina íntegra, em se tratando especificamente do fenômeno de troca de ligantes seja, realmente, o fato da hemoglobina íntegra apresentar uma menor acessibilidade ao solvente aquoso no bolsão hidrofóbico em função da compactação do arranjo quaternário de 144 globinas e 36 cadeias "linkers". Neste caso, haveria apenas uma leve diferenciação no $\mathrm{pH}$ do meio que gera a transição entre espécies no monômero e na hemoglobina íntegra. Esta proposta é pertinente, pois a maior acessibilidade do solvente permitiria, além de um maior contato das moléculas de água com a primeira esfera de coordenação, uma maior interação do heme com os íons hidroxila, ou seja, a maior acessibilidade do solvente no bolsão geraria uma influência mais efetiva do $\mathrm{pH}$ externo na troca de ligantes axiais do heme.

Vale lembrar o trabalho de Hundahl e colaboradores ${ }^{68}$, onde é proposto que as hemoglobinas extracelulares gigantes apresentem menor suscetibilidade a uma mudança conformacional pela solvatação em relação às hemoglobinas tetraméricas, quando da transição entre as formas oxigenada e desoxigenada. Os autores sugerem que devido à elevada massa molecular total e à grande organização, além da menor razão entre a área superficial da hemoglobina íntegra em relação à sua massa total, as chamadas eritrocruorinas apresentam uma maior limitação à acessibilidade da água, o que implicaria em uma menor alteração de estrutura quaternária quando comparada àquela encontrada em hemoglobinas tetraméricas.

É possível supor, portanto, que a troca de ligantes na primeira esfera de coordenação do centro férrico das eritruocruorinas seja menos associada à uma desnaturação propriamente considerada do que em hemoglobinas tetraméricas. De fato, Madura e colaboradores ${ }^{15}$, pesquisando a hemoglobina de L. terrestris, afirmaram que esta hemoproteína apresenta uma mudança conformacional menos intensa que as hemoglobinas tetraméricas, quando ocorre coordenação de ligantes ao centro metálico.

Deste modo, levando-se em conta a similaridade entre as hemoglobinas de L. terrestris e G. paulistus ${ }^{69}$, poder-se-ia inferir que esta característica também se aplica à hemoglobina de $G$. paulistus, o que explicaria o fato das transições entre as espécies formadas na primeira esfera de coordenação do monômero $d$, assim como as condições do meio em que elas ocorrem, apresentarem grande semelhança em relação às transições da hemoglobina íntegra, ressaltando a adequação do monômero $d$ como modelo para a hemoglobina íntegra, especialmente em estudos envolvendo troca de ligantes.

Vale mencionar ainda, que não se conhece perfeitamente a influência dos íons hidroxila neste processo de transição entre espécies. Estudos envolvendo auto-oxidação mostram que, à temperatura ambiente, a conversão de uma espécie hidroxi-meta em hemicromo é muito rápida ${ }^{48}$. Estudos de auto-oxidação em meio alcalino envolvendo a hemoglobina $G$. paulistus corroboram tal assertiva $^{70}$, ou seja, mesmo que ocorra a formação da espécie hidroxi-meta no meio em questão, esta espécie rapidamente se transformaria na espécie hemicromo, inviabilizando sua detecção, nos moldes em que o presente estudo foi desenvolvido, uma vez que o processo de separação cromatográfica já houvera proporcionado a transição aquometa $\rightarrow$ hemicromo. Além disso, vários trabalhos estabelecem que a espécie hidroxi-meta apresentaria, à temperatura ambiente, um equilíbrio térmico entre formas alto e baixo spin, ou seja, uma mistura de configurações alto e baixo spin para a mesma espécie hidroxi-meta ${ }^{71-73}$, o que seria uma dificuldade a mais para sua identificação, principalmente se esta espécie se constituir em apenas um intermediário de configurações mais estáveis. Em verdade, mesmo estudos de ressonância paramagnética eletrônica, como o de Nistor e colaboradores ${ }^{63}$, não identificam a presença da espécie hidroxi-meta.

Por outro lado, uma pequena presença da espécie aquometa foi detectada em pH 7,1, evidenciada através da banda de transferência de carga do ligante para o metal em $630 \mathrm{~nm}$, que é característica desta espécie, indicando que o processo de separação cromatográfica não eliminou totalmente esta espécie, permanecendo em pequena concentração. Isto ratifica, uma vez mais, a complexidade dos equilíbrios químicos formados pelas hemoglobinas em geral, especialmente a hemoglobina de $G$. paulistus, que apresenta no mínimo duas espécies coexistindo em cada $\mathrm{pH}$, visto que as transições não parecem ser totais.

De qualquer maneira, o presente estudo deixa claro que as transições envolvendo mudança de ligantes, que ocorrem na primeira esfera de coordenação do centro férrico, dependem fortemente do $\mathrm{pH}$ do meio. 


\section{Emissão de fluorescência}

Desenvolveu-se um estudo detalhado sobre a emissão de fluorescência dos monômeros $d$ nativo e reconstituído a partir do $\mathrm{pH} 7,0$, analisando-se as intensidades de fluorescência e as posições dos máximos de emissão.

Nas Figuras 3 e 4 estão apresentados os espectros de fluorescência dos monômeros $d$ nativo e reconstituído, respectivamente, notando-se um aumento no máximo de fluorescência em $326 \mathrm{~nm}$ até $\mathrm{pH} 10,3$. Este valor decai acima de $\mathrm{pH}$ 10,3 deslocando o máximo de fluorescência para $336 \mathrm{~nm}$.

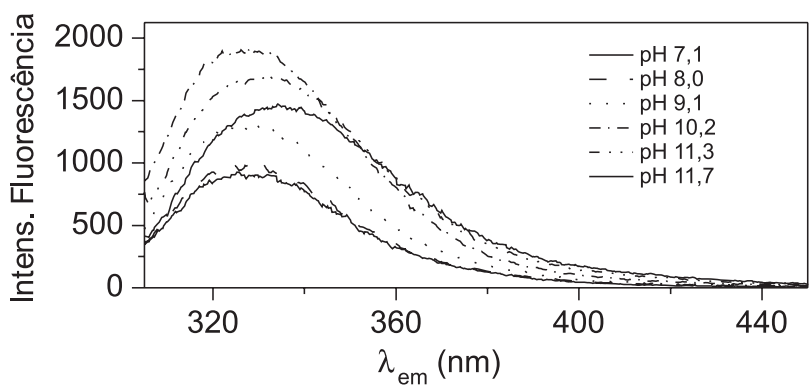

Figura 3. Espectros de emissão de fluorescência do monômero d nativo da metaHb de G. paulistus em função do $\mathrm{pH}$ por titulação, $\lambda_{\text {exc }}=295 \mathrm{~nm}$

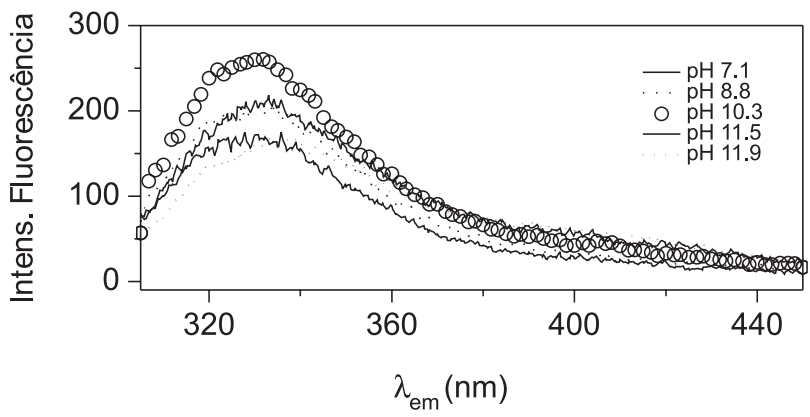

Figura 4. Espectros de emissão de fluorescência do monômero d reconstituído da metaHb de G. paulistus em função do $\mathrm{pH}$ por titulação, $\lambda_{\text {exc }}=295 \mathrm{~nm}$

As Figuras 5 e 6 evidenciam um aumento do rendimento quântico até pH 10,5, sem uma alteração detectável no comprimento de onda do máximo de emissão. Assumindo-se a transferência de energia não-radiante de Förster como o mecanismo de supressão de fluorescência determinante do baixo rendimento quântico de hemoproteínas ${ }^{45,74}$, é possível propor que tal aumento de intensidade de fluorescência se deva a um processo inicial de desenovelamento polipeptídico. Esse desenovelamento inicial, devido à alteração do $\mathrm{pH}$ do meio, possivelmente afasta e/ou altera as orientações relativas dos indóis dos triptofanos em relação ao heme, que é o principal supressor de fluorescência nas hemoproteínas, diminuindo a supressão e aumentando o rendimento quântico. Apesar desse desenovelamento aumentar o rendimento quântico, não ocorre alteração do comprimento de onda de emissão, o que indica que a constante dielétrica nas circunvizinhanças dos triptofanos permanece a mesma, ou seja, apesar do desenovelamento inicial, não ocorre um aumento significativo do contato do solvente aquoso com os triptofanos, fato este que aumentaria a supressão dinâmica da fluorescência.

Entretanto, após o pH 10,5, ocorre não somente uma diminuição do rendimento quântico como também um deslocamento batocrômico do máximo de emissão. Estes dois fenômenos analisados em con-

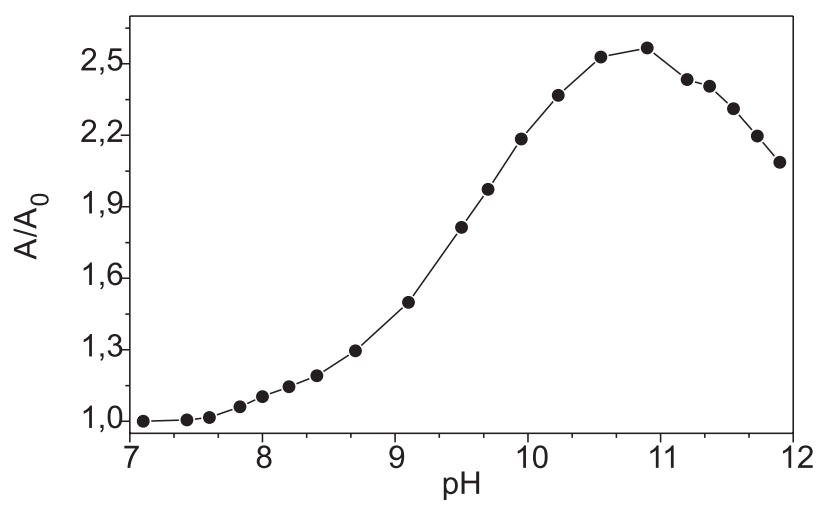

Figura 5. Áreas normalizadas dos espectros de emissão de fluorescência do monômero d nativo da metaHb de G. paulistus, $\lambda_{\text {exc }}=295 \mathrm{~nm}, \lambda_{\text {em }}=305-450$ $n m$, em função do $\mathrm{pH}$

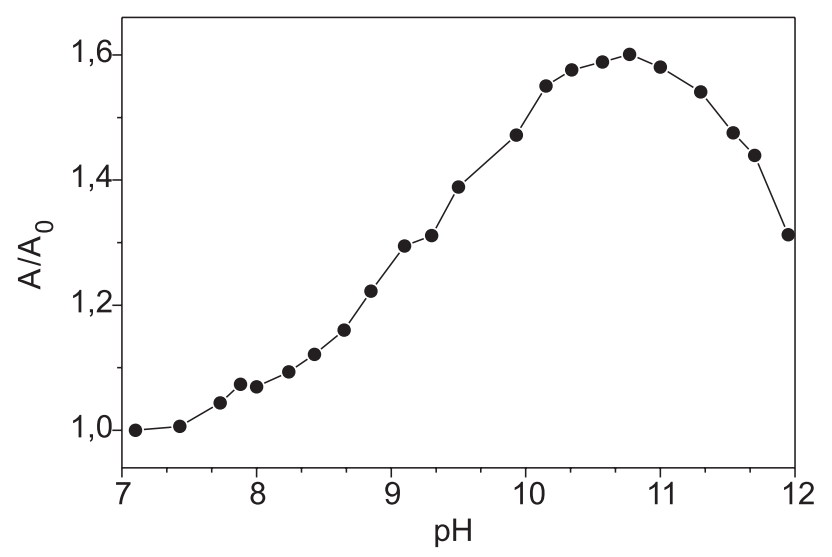

Figura 6. Áreas normalizadas dos espectros de emissão de fluorescência do monômero d reconstituído da metaHb de G. paulistus, $\lambda_{e x c}=295 \mathrm{~nm}, \lambda_{e m}=$ 305-450 nm, em função do $\mathrm{pH}$

junto indicam, agora sim, um contato mais efetivo do solvente nos resíduos de triptofano. Portanto, a supressão dinâmica da fluorescência tornou-se maior, assim como a estabilização dos orbitais $\pi^{*}$ gerou o maior deslocamento de Stokes observado. De fato, como os orbitais $\pi^{*}$ são mais externos, e, portanto, mais suscetíveis às interações com o solvente que os orbitais $\pi$ ligantes, uma estabilização bem mais significativa tende a ocorrer com os orbitais $\pi^{*}$, fazendo com que a transição em questão se torne menos energética e, por conseguinte, com um maior comprimento de onda.

O comportamento fluorescente observado, tanto para o monômero nativo como para o reconstituído, apresenta similaridade com o perfil fluorescente da hemoglobina íntegra ${ }^{75,76}$, indicando, assim como constatado através da espectroscopia de absorção na região do ultravioleta-visível, que o monômero $d$ se constitui em um excelente sistema modelo para o estudo de certas propriedades da hemoglobina íntegra, como por ex., a troca de ligantes.

\section{CONCLUSÕES}

O presente trabalho evidencia que as trocas de ligantes que ocorrem na primeira esfera de coordenação em meio alcalino dos monômeros $d$ nativo e reconstituído da hemoglobina extracelular gigante de $G$. paulistus apresentam uma forte dependência do $\mathrm{pH}$. Foram identificadas duas espécies hexacoordenadas, que são as formas aquometa e hemicromo, assim como a espécie pentacoordenada, cujo quinto ligante seria o grupo imidazolato da histidina proximal, o que difere este pentacoordenado daquele encontrado 
em meio ácido, cujo imidazol histidínico não está desprotonado ${ }^{23}$. A identificação da espécie pentacoordenada é efetuada através do deslocamento hipsocrômico da banda de Soret e da diminuição da absortividade molar desta mesma banda, além da ocorrência da banda TCLM em comprimento de onda característico de configurações eletrônicas alto spin.

Os estudos de fluorescência do monômero $d$ nativo da hemoglobina de G. paulistus mostraram que, a partir de $\mathrm{pH} 7,0$, a alcalinização promove um aumento no rendimento quântico, indicando um possível afastamento e/ou mudança nas respectivas orientações dos resíduos de triptofano em relação ao heme, que é o principal supressor de fluorescência de hemoproteínas, conforme o mecanismo de Förster. Esta intensificação ocorre até um pH em torno de 10,0 e acima deste valor observa-se uma queda do rendimento quântico juntamente a um deslocamento do máximo de emissão de 326 para $336 \mathrm{~nm}$. Tanto o aumento do comprimento de onda de emissão como a diminuição da intensidade de fluorescência indicam um contato mais efetivo do solvente aquoso com os resíduos de triptofano. Tal fenômeno ocorre em valor próximo ao $\mathrm{pH}$ em que acontece a transição do hexacoordenado para pentacoordenado, o que sugere a importância da maior acessibilidade do solvente aquoso nesta transição da primeira esfera de coordenação do centro férrico. Portanto, é possível inferir que o surgimento da espécie pentacoordenada esteja correlacionado com um desenovelamento gradual e dependente do $\mathrm{pH}$ da cadeia monomérica $d$.

O monômero $d$ reconstituído da hemoglobina de G. paulistus mostrou um comportamento espectroscópico semelhante ao apresentado pelo monômero $d$ nativo, com relação tanto à fluorescência como aos espectros de absorção na região do ultravioleta-visível. Estes resultados são indícios de que a estrutura do monômero $d$ reconstituído é similar àquela do monômero $d$ nativo. Outros estudos espectroscópicos estão em progresso para melhor avaliação dessa semelhança e suas implicações estruturais.

\section{AGRADECIMENTOS}

A D. R. Cardoso pelas valiosas discussões a respeito dos desdobramentos de orbitais moleculares de complexos porfirínicos e hemoproteínas e suas implicações espectroscópicas; ao Prof. Dr. M. Tabak por todo o apoio fornecido para viabilizar a submissão deste trabalho; à CAPES pela bolsa de mestrado proporcionada a J. C. Ribelatto e ao CNPq pela bolsa de doutorado concedida a L. M. Moreira.

\section{REFERÊNCIAS}

1. Marques, M. B.; Meirelles, N. C.; Comp. Biochem. Physiol., Part B: Biochem. Mol. Biol. 1995, 111, 311.

2. Righi, G.; Monitore Zool. Ital.(N.S) 1972, 6, 19.

3. Rossi, J. -P.; Nuutinen, V.; Appl. Soil Ecol. 2004, 27, 189.

4. Costa, M. C. P.; Bonafé, C. F. S.; Meireles, N. C.; Galembeck, F.; Braz. J. Med. Res. 1988, 21, 115.

5. Meirelles, N. C.; Oliveira, B.; Oliveira, A. R.; de Paula, E.; Marangoni, S.; Rennebeck, G. M.; Comp. Biochem. Physiol., Part A: Mol. Integr. Physiol. 1987, 88A, 337.

6. Vinogradov, S. N.; Micron 2004, 35, 127.

7. Martin, P. D.; Kuchumov, A. R.; Green, B. R.; J. Mol. Biol. 1996, 255, 154.

8. Kuchumov, A. R.; Taveau, J. C.; Lamy, J. N.; Wall, J. S.; Weber, R. E.; Vinogradov, S. N.; J. Mol. Biol. 1998, 289, 1361.

9. Viana, E.; Silva, C. H. T. P.; Tabak, M.; Imasato, H.; Garrat, R. C.; Biochim. Biophys. Acta 1998, 1383, 130.

10. Kapp, O. H.; Polidori, G.; Mainwaring, M. G.; Crewe, A. V.; Vinogradov, S. N.; J. Biol. Chem. 1984, 259, 628.

11. Haas, F.; Kuchumov, A.; Taveau, J. C.; Boisset, N.; Vinogradov, S. N.; Lamy, J. N.; Biochemistry 1997, 36, 7330.
12. Mouche, F., Boisset, N.; Penczek, P. A.; J. Struct. Biol. 2001, 133, 176.

13. Krebs, A.; Vinogradov, S. N.; Zipper, P.; Biopolymers 1998, 45, 289.

14. Daniel, E.; Lustig, A.; David, M. M.; Tsfadia, Y.; Micron 2004, 35, 131.

15. Madura, J. D.; Salter, E. A.; Wierzbicki, A.; Dalal, P.; Harrington, J. P.; J. Mol. Struct. 2002, 592, 173.

16. Bailly, X.; Vinogradov, S.; J. Inorg. Biochem. 2005, 99, 142.

17. Hirsch, R. E.; Jelicks, L. A.; Wittenberg, B. A.; Kaul, D. K.; Shear, H. L.; Harrington, J. P.; Art. Cells, Blood Subs., And Immob. Biotech. 1997, 25, 429.

18. Dorman, S. C.; Harrington, J. P.; Martin, M. S.; Johnson, T. V.; J. Inorg. Biochem. 2004, 98, 185.

19. Strand, K.; Knapp, J. E.; Bhyravbhatia, B.; Royer Jr., W. E.; J. Mol. Biol. 2004, 344, 119

20. Silva, J. L.; Villas-Boas, M.; Bonafe, C. F. S.; Meirelles, N. C.; J. Biol. Chem. 1989, 264, 15863.

21. Royer, W. E.; Strand, K.; Van Hell, M.; Hendrickson, W. A.; Proc. Natl. Acad. Sci. U. S. A. 2000, 97, 7107.

22. Royer, W. E.; Knapp, J. E.; Strand, K.; Heaslet, H. A.; Trends Biochem. Sci. 2001, 26, 297.

23. Ribelatto, J. C.; Poli, A. L.; Moreira, L. M.; Imasato, H.; Quim. Nova 2005 , no prelo.

24. Marques, H. M.; Brown, K. L.; Coord. Chem. Rev. 2002, 225, 123.

25. Dasgupta, S.; Rousseau, D. L.; Anni, H.; Yonetani, T.; J. Biol. Chem. 1989, $264,654$.

26. Trent III, J. T.; Hvitved, A. N.; Hargrove, M. S.; Biochemistry 2001, 40, 6155.

27. Sage, J. T.; Morikis, D.; Champion, P. M.; Biochemistry 1991, 30, 1227.

28. Palaniappan, V.; Bocian, D. F.; Biochemistry 1994, 33, 14264.

29. Longa, S. D.; Amiconi, G.; Salah, O. A.; Ascone, I.; Barteri, M.; Bertollini, A.; Bianconi, A.; Castellano, A. C.; Biochim. Biophys. Acta 1996, 1294, 72.

30. Verde, C.; Howes, B. D.; de Rosa, M. C.; Raiola. L.; Smulevich, G.; Williams, R.; Giardina, B.; Parisi, E.; Di Prisco, G.; Protein Sci. 2004, 13, 2766.

31. Robinson, V. L.; Smith, B. B.; Arnone, A.; Biochemitry 2003, 42, 10113.

32. Pesce, A.; Dewilde, S.; Nardini, M.; Moens, L.; Ascenzi, P.; Hankeln, T.; Burmester, T.; Bolognesi, M.; Mícron 2004, 35, 63.

33. Das, T. K.; Boffi, A.; Chiancone, E.; Rousseau, D. L.; J. Biol. Chem. 1999, 274, 2916.

34. Baron, C. P.; Skibsted, L. H.; Andersen, H. J.; Free Radical Biol. Med. 2000, 28, 549 .

35. Smith, D. M. A.; Dupuis, M.; Vorpagel, E. R.; Straatsma, T. P.; J. Am. Chem. Soc. 2003, 125, 2711.

36. Medakovic, V.; Zaric, S. D.; Inorg. Chim. Acta 2003, 349, 1.

37. Zaric, S. D.; Popovic, D. M.; Knapp, E.; Biochemistry 2001, 40, 7914.

38. Fahnenschmidt, M.; Bittl, R.; Rau, H. K.; Haehnel, W.; Lubitz, W.; Chem. Phys. Lett. 2000, 323, 329.

39. Walker, F. A.; Coord. Chem. Rev. 1999, 185-186, 471.

40. Moreira, L. M.; Ribelatto, J. C.; Imasato, H.; Quim. Nova 2004, $27,958$.

41. Imasato, H.; Tinto, M. H.; Perussi, J. R.; Tabak, M.; Comp. Biochem. Physiol., Part B: Biochem. Mol. Biol. 1995, 112, 217.

42. Zhu, H.; Ownby, D. W.; Rigs, C. K.; Nolasco, N. J.; Stoops, J. K.; Riggs, A. F.; J. Biol. Chem. 1996, 271, 30007.

43. Teale, F. W. J.; Biochim. Biophys. Acta 1959, 35, 543.

44. Perczel, A.; Park, K.; Fasman, G. D.; Anal. Biochem. 1992, 203, 83.

45. Antonini, E.; Rossi-Bernardi, L.; Chiancone, E.; Methods in Enzymology, $1^{\text {st }}$ ed.; Academic Press, 1981.

46. Vitagliano, L.; Bonomi, G.; Riccio, A.; Prisco, G. D.; Smulevich, G.; Mazzarella, L.; Eur. J. Biochem. 2004, 271, 1651.

47. Rachmilewitz, E. A.; Peisach, J.; Blumberg, W. E.; J. Biol. Chem. 1971, $246,3356$.

48. Tsuruga, M.; Matsuoka, A.; Hachimori, A.; Sugawara, Y.; Shikama, K.; J. Biol. Chem. 1998, 273, 8607.

49. Visca, P.; Fabozzi, G.; Petrucca, A.; Ciaccio, C.; Coletta, M.; De Sanctis, G.; Bolognesi, M.; Milani, M.; Ascenzi, P.; Biochem. Biophys. Res. Commun. 2002, 294, 1064.

50. Gilles-Gonzalez, M. A.; Gonzalez, G.; Perutz, M. F.; Biochemistry 1994, 33, 8067.

51. Quillin, M. L.; Arduini, R. M.; Olson, J. S.; Phillips Jr., G. N.; J. Mol. Biol. 1993, 234, 140.

52. Tada, T.; Watanabe, Y.; Matsuoka, A.; Ikeda-Saito, M.; Imai, K.; Nihei, Y.; Shikama, K.; Biochim. Biophys. Acta 1998, 1387, 165.

53. Kamimura, S.; Matsuoka, A.; Imai, K.; Shikama, K.; Eur. J. Biochem. 2003, $270,1424$.

54. Matsuoka, A.; Kobayashi, N.; Shikama, K.; Eur. J. Biochem. 1992, 210, 337.

55. Sigman, J. A.; Pond, A. E.; Dawson, J. H.; Lu, Y.; Biochemistry 1999, 38, 11122. 
56. Rajarathnam, K.; La Mar, G. N.; Chiu, M. L.; Sligar, S. G.; Singh, J. P.; Smith, K. M.; J. Am. Chem. Soc. 1991, 113, 7886.

57. Morikis, D.; Champion, P. M.; Springer, B. A.; Egeberg, K. D.; Sligar, S G.; J. Biol. Chem. 1990, 265, 12143.

58. Bujons, J.; Dikiy, A.; Ferrer, J. C.; Banci, L.; Mauk, A. G.; Eur. J. Biochem 1997, 243, 72.

59. Smulevich, G.; Paoli, M.; De Sanctis, G.; Mantini, A. R.; Ascoli, F.; Biochemistry 1997, 36, 640.

60. Wright, P. J.; English, A. N.; J. Biol. Inorg. Chem. 2001, 6, 348.

61. Cheek, J.; Low, D. W.; Gray, H. B.; Dawson, J. H.; Biochem. Biophys. Res. Commun. 1998, 253, 195.

62. Harris, D.; Loew, G.; J. Am. Chem. Soc. 1993, 115, 5799.

63. Nistor, S. V.; Goovaerts, E.; Van Doorslaer, S.; Dewilde, S.; Moens, L.; Chem. Phys. Lett. 2002, 361, 355.

64. Boffi, A.; Guarrera, L.; Giangiacomo, L.; Spagnuolo, C.; Chiancone, E.; Biochemistry 2000, 39, 3500.

65. Riccio, A.; Vitagliano, L.; Prisco, G. D.; Zagari, A.; Mazzarela, L.; Proc. Natl. Acad. Sci U. S. A. 2002, 99, 9801.

66. Paoli, M.; Dodson, G.; Liddington, R. C.; Wilkinson, A. J.; J. Mol. Biol. 1997, 271, 161 .
67. Agustinho, S. C. M.; Tinto, M. H.; Imasato, H.; Tominaga, T. T.; Perussi, J. R.; Tabak, M.; Biochim. Biophys. Acta 1996, 1298, 148.

68. Hundahl, C.; Fago, A.; Weber, R. E.; Comp. Biochem. Physiol., Part B: Biochem. Mol. Biol. 2003, 136, 83.

69. Cabral, C. B.; Imasato, H.; Rosa, J. C.; Laure, H. J.; Silva, C. H. T. P.; Tabak, M.; Garrat, R. C.; Green, L. J.; Biophys. Chem. 2002, 97, 139.

70. Poli, A. L.; Moreira, L. M.; Hidalgo, A. A.; Imasato, H.; Biophys. Chem. 2005, 114, 253.

71. Longa, S. D.; Pin, S.; Cortès, R.; Soldatov, A. V.; Alpert, B.; Biophys. J. 1998, 75, 3154

72. Levy, A.; Kuppusamy, P.; Rifkind, J. M.; Biochemistry 1990, 29, 9311.

73. Rousseau, D. L.; Ching, Y.; Brunori, M.; Giacometti, G. M.; J. Biol. Chem. 1989, 264, 7878

74. Lakowicz, J.; Principles of fluorescence spectroscopy, $1^{\text {st }}$ ed., Plenum Press: New York, 1983.

75. Imasato, H.; Tinto, M. H.; Perussi, J. R.; Tabak, M.; Comp. Biochem. Physiol., Part B: Biochem. Mol. Biol. 1995, 112, 217.

76. Perussi, J. R.; Imasato, H.; Tabak, M.; Meirelles, N. C.; Physiol. Chem. Physics Med. NMR 1990, 22, 159. 